\title{
Modelling of the Effect of a Thin Stiffener on the Boundary of a Nonlinear Thermoelastic Plate
}

\section{Rahmani}

University of Tizi-ouzou

Faculté des sciences, département de mathématiques, université de Tiziouzou, Algeria

E-mail: rahmani_lei@yahoo.fr

Received April 4, 2008; revised October 29, 2008; published online July 15, 2009

\begin{abstract}
We consider a dynamic nonlinear model for a heterogeneous thermoelastic plate consisting of a thin highly rigid body of high thermal conductivity perfectly glued on a portion of the boundary of an elastic plate. This model, which describes the nonlinear oscillations of a plate subjected to thermal effects is referred to as the "full von Karman thermoelastic system". Our aim is to model this junction and reproduce the effect of the thin body by means of approximate boundary conditions, obtained by an asymptotic analysis with respect to the thickness of this body.
\end{abstract}

Key words: Ventcel's conditions, von Karman thermoelastic system, thin plate, stiffener, multi-structure, asymptotic analysis.

\section{Introduction}

In this paper, we consider a dynamic nonlinear model for a heterogeneous thermoelastic plate consisting of a thin highly rigid body of high thermal conductivity perfectly glued on a portion of the boundary of an elastic plate. Our aim is to simulate mathematically this junction and reproduce the effect of the thin body (which may be viewed as a stiffener) by means of approximate boundary conditions on the junction region. To this end, we examine the asymptotic behavior of the solution as the thickness $\delta$ of the rigid body goes to zero. This analysis leads to a nonstandard boundary value problem in which the boundary conditions involve tangential and time derivatives of order equal to that of the interior differential operator. This type of boundary condition is called Ventcel's condition $[2,8]$.

The main motivation of the present work comes from numerical considerations. It lies in the fact that the thickness of the inserted body appears to be very small with respect to the dimensions of the plate: in such a situation, the discretisation inside the domain occupied by the stiffener needs very thin meshes and may excessively increase the computational costs. Moreover an 
excessive overmeshing may induce some numerical locking effects which can damage the approximating properties of the numerical scheme. An alternative approach consists in deriving approximate boundary conditions, incorporating in an approximate way the effect of the thin body. More precisely, we seek an approximate problem posed over the domain of the plate (i.e., not including the stiffener), but taking into account its effect via new boundary conditions.

The idea of introducing this kind of boundary conditions is largely used in modelling problems involving thin layers. It was proposed for a long time to incorporate the effect of a thin coating in the scattering of electromagnetic waves by a perfectly conducting obstacle (see, e.g. [1, 4, 5]). It has been also investigated by several authors problems of reinforcement by thin layers in structural mechanics, (see $[9,10,11,13]$ ).

The study of the present paper extends the results obtained by the author in [10], where a non linear model which does not account for the thermal effects was considered.

The paper is organized as follows: after giving the variational formulation of the problem, we use a scaling argument to put the problem obtained when the thickness of the rigid body is varying in a common functional setting. Then, we establish a priori estimates which allow us to extract a weakly convergent subsequence. We then identify the variational problem solved by the weak limit and find the Ventcel model. Finally, we mention that it is important to test if the obtained solution of the limit problem is unique. It is well known that such a result provides the convergence of the whole sequence and thus one may analyze without ambiguity the proximity of solutions of both models as $\delta \rightarrow 0$. However, this question is delicate and it remains to be investigated.

\section{Statement of the Problem}

In order to state the problem, consider $\Omega_{+} \subset R^{2}$, an open bounded set. We suppose that $\partial \Omega_{+}=\bar{\Gamma}_{+} \cup \bar{\Sigma}$ and $\Gamma_{+} \cap \Sigma=\emptyset$. Let $\nu=\left(\nu_{1}, \nu_{2}\right)$ be the inner unit normal to $\Sigma$ and $\tau$ be the tangent unit vector field to $\Sigma$ such that the basis $(\tau, \nu)$ is direct in each point of $\Sigma$. Denote by $s$ a curvilinear abscissa (arc length) along $\Sigma$ oriented according to $\tau$ and consider

$$
\Omega_{-}^{\delta}=\{s+y \nu ; \quad s \in \Sigma ; \quad 0<y<\delta\} .
$$

We set $\Sigma_{-}^{\delta}=\{s+\delta \nu ; s \in \Sigma\} ; \partial \Omega_{-}^{\delta}=\Gamma_{-}^{\delta} \cup \Sigma \cup \Sigma_{-}^{\delta}$ with $\Gamma_{-}^{\delta} \cap \Sigma=\emptyset$ and $\Omega^{\delta}=\Omega_{+} \cup \Sigma \cup \Omega_{-}^{\delta}$. The set $\Omega^{\delta}$ is thus the domain occupied by a bidimensional elastic plate comprising two substructures "perfectly bonded" together along their common boundary $\Sigma$. In what follows, the functions $w$ and $u=\left(u_{1}, u_{2}\right)$ represent respectively, the deflexion and the in-plane displacement of the plate, while $\theta$ and $\phi$ describe the temperature affecting the vertical displacement and the horizontal (in-plane) displacement, respectively.

The full von Karman thermoelastic system of the plate reads $[3,7,6]$ :

$$
\rho u^{\prime \prime}-\operatorname{div}[C(\varepsilon(u)+f(\nabla w))]+\lambda \nabla \phi=0 \quad \text { in } \Omega^{\delta} \times(0, T),
$$




$$
\begin{aligned}
& \rho[I-\Delta] w^{\prime \prime}+D \Delta^{2} w-\operatorname{div}[C(\varepsilon(u)+f(\nabla w)) \nabla w]+\lambda \Delta \theta=0 \text { in } \Omega^{\delta} \times(0, T), \\
& \rho \phi^{\prime}-k \Delta \phi+\lambda \operatorname{div} u^{\prime}=0 \quad \text { in } \Omega^{\delta} \times(0, T), \\
& \rho \theta^{\prime}-k \Delta \theta-\lambda \Delta w^{\prime}=0 \quad \text { in } \Omega^{\delta} \times(0, T),
\end{aligned}
$$

with Dirichlet (clamped) conditions on the portion of the boundary $\Gamma_{+} \cup \Gamma_{-}^{\delta}$

$$
u=0, \quad w=\partial_{\nu} w=0, \quad \theta=0, \quad \phi=0 \quad \text { on } \quad \Gamma_{+} \cup \Gamma_{-}^{\delta} \times(0, T),
$$

and free boundary conditions on the boundary $\Sigma_{-}^{\delta} \times(0, T)$ :

$$
\begin{aligned}
& C[\varepsilon(u)+f(\nabla w)] \nu=0, \quad D\left[\Delta w+(1-\mu) B_{1} w\right]=0, \\
& D\left[\partial_{\nu} \Delta w+(1-\mu) \partial_{s} B_{2} w\right]-\rho \partial_{\nu} w^{\prime \prime}-C[\varepsilon(u)+f(\nabla w)] \nu \cdot \nabla w+\lambda \partial_{\nu} \theta=0, \\
& k \partial_{\nu} \theta+\lambda \partial_{\nu} w^{\prime}=0, \quad k \partial_{\nu} \phi-\lambda u^{\prime} \nu=0 .
\end{aligned}
$$

We define also the transmission conditions on $\Sigma \times(0, T)$ by

$$
\begin{aligned}
& {[[u]]=0, \quad[[w]]=\left[\left[\partial_{\nu} w\right]\right]=0, \quad[[\theta]]=[[\phi]]=0,} \\
& {[[C[\varepsilon(u)+f(\nabla w)] \nu]]=0, \quad\left[\left[D\left[\Delta w+(1-\mu) B_{1} w\right]\right]\right]=0,} \\
& {\left[\left[k \partial_{\nu} \theta+\lambda \partial_{\nu} w^{\prime}\right]\right]=0, \quad\left[\left[k \partial_{\nu} \phi-\lambda u^{\prime} \nu\right]\right]=0,} \\
& {\left[\left[D\left[\partial_{\nu} \Delta w+(1-\mu) \partial_{s} B_{2} w\right]-\rho \partial_{\nu} w^{\prime \prime}-C[\varepsilon(u)+f(\nabla w)] \nu \nabla w+\lambda \partial_{\nu} \theta\right]\right]=0 .}
\end{aligned}
$$

With (2.1) and (2.4) we associate the initial conditions given in $\Omega^{\delta}$ :

$$
u(0)=u_{0}, u^{\prime}(0)=u_{1}, w(0)=w_{0}, w^{\prime}(0)=w_{1}, \theta(0)=\theta_{0}, \phi(0)=\phi_{0} .
$$

By [[]] we denote the jump through $\Sigma$ of a function or distribution defined on $\Omega^{\delta}$ that admits in some sense traces on $\Sigma$. The fourth order tensor $C$ belongs to $S$, the space of $2 \times 2$ symmetric matrices, and it is defined by

$$
C(\zeta)=\frac{E}{\left(1-\mu^{2}\right)}\left[\mu(\operatorname{tr} \zeta) I_{S}+(1-\mu) \zeta\right], \quad \forall \zeta \in S,
$$

where $I_{S}$ is the identity matrix and $(\operatorname{tr} \zeta)$ is the trace of $\zeta$. Moreover, the strain tensor is given by $\epsilon(u)=1 / 2\left(\nabla u+\nabla^{T} u\right)$. The function $f$ is given by $f(s)=(1 / 2) s \otimes s, \forall s \in \mathbb{R}^{2}$ and the boundary operators are defined by

$B_{1} w \equiv 2 \nu_{1} \nu_{2} \partial_{x y}^{2} w-\nu_{1}^{2} \partial_{y}^{2} w-\nu_{2}^{2} \partial_{x}^{2} w, \quad B_{2} w \equiv\left(\nu_{1}^{2}-\nu_{2}^{2}\right) \partial_{x y}^{2} w+\nu_{1} \nu_{2}\left(\partial_{y}^{2} w-\partial_{x}^{2} w\right)$.

$D=\frac{E}{\left(1-\mu^{2}\right)}$ represents the flexural rigidity of the plate; $E$ is the Young's modulus, $\mu$ is the Poisson ratio of the material and $\rho$ is its mass density. $k$ is the coefficient of thermal conductivity and $\lambda=D \alpha(1+\mu) / 2$, where $\alpha$ denotes the coefficient of thermal expansion. We assume that $E>0,0<\mu<\frac{1}{2}$ and that the coefficients described above are piecewise constant: $E=E_{+}$in $\Omega_{+}$ and $E=E_{-} / \delta$ in $\Omega_{-}^{\delta}, \mu=\mu_{+}$in $\Omega_{+}$and $\mu=\mu_{-}$in $\Omega_{-}^{\delta} ; \rho=\rho_{+}$in $\Omega_{+}$ and $\rho=\rho_{-} / \delta$ in $\Omega_{-}^{\delta} ; k=k_{+}$in $\Omega_{+}$and $k=k_{-} / \delta$ in $\Omega_{-}^{\delta} ; \alpha=\alpha_{+}$in $\Omega_{+}$ and $\alpha=\alpha_{-}$in $\Omega_{-}^{\delta}$, where $E_{+}, E_{-}, \mu_{+}, \mu_{-}, \rho_{+}, \rho_{-}, k_{+}, k_{-}, \alpha_{+}$and $\alpha_{-}$are constants independent of $\delta$. As a consequence, $D$ and $\lambda$ are independent of $\delta$ in $\Omega_{+}$and vary as $\delta^{-1}$ in $\Omega_{-}^{\delta}\left(D=D_{+}\right.$in $\Omega_{+}$and $\delta^{-1} D_{-}$in $\Omega_{-}^{\delta} ; \lambda=\lambda_{+}$in $\Omega_{+}$ and $\delta^{-1} D_{-}$in $\Omega_{-}^{\delta}$ ). We also denote by $g^{\prime}, g^{\prime \prime}$ the time derivatives of a function $g$. 
Remark 1. The derivation of the above model parallels the arguments presented in [6] for linear thermoelastic equations after incorporating the nonlinear strain displacement relations. This model is described in [3] and [7], up a normalization of the physical constants.

The above system describes the nonlinear oscillations of a plate subjected to thermal effects and accounting for vertical and in-plane accelerations. The resulting model consists of an elastodynamic system coupled with the Kirchhoff plate equation and two heat equations. As such, it is referred to as the "full von Karman thermoelastic system". In our case, the structure considered consists of a thin plate with a stiffener glued on a portion of its boundary. These two elastic bodies are perfectly "bonded" along their common boundary $\Sigma$, thus forming together an elastic multi-structure, viewed as an elastic bidimensional plate occupying the domain $\Omega^{\delta}$. This plate is clamped along its boundary $\Gamma_{+} \cup \Gamma_{-}^{\delta}$ and is motion free on its exterior boundary $\Sigma_{-}^{\delta}$. The assumption on the Young's modulus, the mass density and the thermal conductivity (they are independent of $\delta$ in $\Omega_{+}$and vary as $\delta^{-1}$ in $\Omega_{-}^{\delta}$ ) express that the elastic material constituting the stiffener must be "more rigid", "heavier" and more "conductive" than that constituting the plate, in such a way that it compensates for the thickness.

The relations along $\Sigma$, which formally express the continuity along the common portion of the two boundaries are called transmission conditions. The condition $[[u]]=0,[[w]]=\left[\left[\partial_{\nu} w\right]\right]=0$ along $\Sigma$ shows in particular that we are modelling a situation where the inserted portion of the thin body is "perfectly bonded" to the plate, thus we exclude situations where the inserted portion could slide along or part away from the plate.

Remark 2. The assumption made on the physical coefficients of the stiffener (they behave as $\delta^{-1}$ ) needs to be assumed in order to obtain the limit model described in this paper and that takes into account the thermal and mechanical effect of the stiffener. Other ratios between the asymptotic orders in the plate and the body can lead to different limit behaviors. Indeed, the case of the thermoelastic Von Karman system with constant physical coefficients have been studied by the author for a plate surrounded with a thin layer, via a different method (see [12]). The limit model obtained does not take into account the presence of the thin layer. Other choices can be made: coefficients varying as $\delta^{-a}, a \in \mathbb{R}_{+}^{*}$ in the stiffener, thermal coefficients independents of $\delta$ and mechanical coefficients varying as $\delta^{-1}, \ldots$. Each of these questions requires a full study in order to identify the limit problem it leads to. We can refer to [9], where the case of coefficients varying as $\delta^{-a}$ have been studied for the Kirchhoff-love plate equations.

In what follows, we recall that the symbol $\langle\cdot, \cdot\rangle_{\Omega}$ represents the inner product in $\left[L^{2}(\Omega)\right]^{k}, k \in N$. Let $\{u, w, \phi, \theta\}$ be a classical solution of $(2.1)-(2.4)$. The total energy of the plate is given by

$$
\begin{aligned}
& E(t)=\frac{1}{2}\left\{\rho\left\|u^{\prime}(t)\right\|_{L^{2}(\Omega)}^{2}+\rho\left\|w^{\prime}(t)\right\|_{L^{2}(\Omega)}^{2}+\rho\left\|\nabla w^{\prime}(t)\right\|_{L^{2}(\Omega)}^{2}+a(w(t), w(t))\right. \\
& \left.+\rho\|\theta(t)\|_{L^{2}(\Omega)}^{2}+\rho\|\phi(t)\|_{L^{2}(\Omega)}^{2}+\langle C[\epsilon(u(t))+f(\nabla w(t))], \epsilon(u(t))+f(\nabla w(t))\rangle_{\Omega^{\delta}}\right\}
\end{aligned}
$$


where

$$
\begin{aligned}
a(w, \psi) & =\int_{\Omega^{\delta}} D\left\{\left(\partial_{x}^{2} w+\mu \partial_{y}^{2} w\right) \partial_{x}^{2} \psi+2(1-\mu) \partial_{x y}^{2} w \partial_{x y}^{2} \psi\right. \\
& \left.+\left(\partial_{y}^{2} w+\mu \partial_{x}^{2} w\right) \partial_{y}^{2} \psi\right\} d \Omega^{\delta}
\end{aligned}
$$

Let us introduce the following functional spaces

$$
W\left(\Omega^{\delta}\right)=\left\{w \in H^{2}\left(\Omega^{\delta}\right) ; w_{\mid \Gamma_{+} \cup \Gamma_{-}^{\delta}}=\partial_{\nu} w_{\mid \Gamma_{+} \cup \Gamma_{-}^{\delta}}=0\right\},
$$$$
V\left(\Omega^{\delta}\right)=\left\{w \in H^{1}\left(\Omega^{\delta}\right) ; w_{\mid \Gamma_{+} \cup \Gamma_{-}^{\delta}}=0\right\}, U\left(\Omega^{\delta}\right)=\left\{u \in\left(H^{1}\left(\Omega^{\delta}\right)\right)^{2} ; u_{\mid \Gamma_{+} \cup \Gamma_{-}^{\delta}}=0\right\} .
$$

Let us take the inner product in $\left[L^{2}\left(\Omega^{\delta}\right)\right]^{2}$ of $(2.1),(2.2),(2.3)$ and $(2.4)$ respectively with $\varphi \in U\left(\Omega^{\delta}\right), \psi \in W\left(\Omega^{\delta}\right), \zeta \in V\left(\Omega^{\delta}\right)$ and $\eta \in V\left(\Omega^{\delta}\right)$, by using (2.1)-(2.6) we get

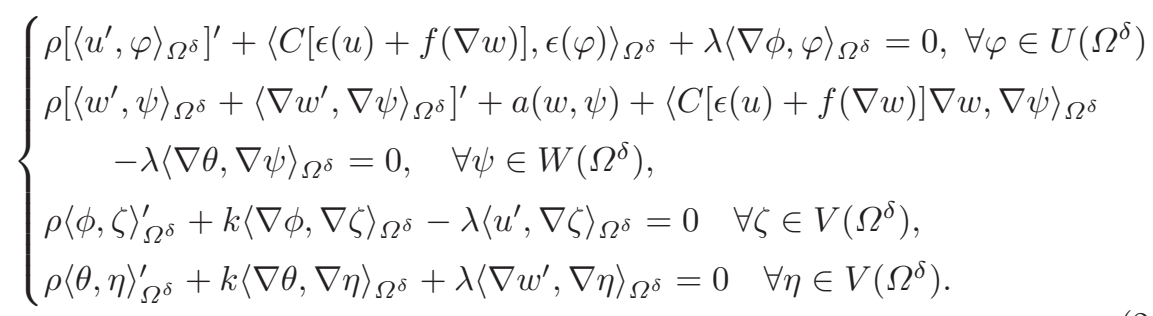

Definition 1. We say that $\{u, w, \phi, \theta\}$ is a weak solution of (2.1)-(2.7) if

$$
\begin{aligned}
& u \in L^{\infty}\left(0, T ; U\left(\Omega^{\delta}\right)\right), \quad u^{\prime} \in L^{\infty}\left(0, T ;\left(L^{2}\left(\Omega^{\delta}\right)\right)^{2}\right) \\
& w \in L^{\infty}\left(0, T ; W\left(\Omega^{\delta}\right)\right), w^{\prime} \in L^{\infty}\left(0, T ; V\left(\Omega^{\delta}\right)\right), \\
& \phi, \theta \in L^{\infty}\left(0, T ; L^{2}\left(\Omega^{\delta}\right)\right) \cap L^{2}\left(0, T ; V\left(\Omega^{\delta}\right)\right)
\end{aligned}
$$

and satisfy (2.7) and (2.8).

Existence of weak solutions of von Karman thermoelastic system is well known and its proof is rather standard. It relies on the application of the nonlinear Galerkin method (see [3, 7]). However, the proof of the uniqueness is much more delicate. We can refer to $[3,7]$ for a rigorous justification of the uniqueness of the weak solution of the von Karman thermoelastic system.

\section{The Scaled Problem}

Since our objective is to study the behavior of the solution of the problem (2.8) as $\delta \rightarrow 0$ and because this last one is defined on the set $\Omega^{\delta}$ which itself varies with $\delta$, our first task naturally consists in transforming the problem into a problem set on a domain that does not depend on $\delta$. Hence, this section is devoted to a change of scaling along the thickness of the rigid body, leading to a formulation having a common functional setting as $\delta$ is varying. 


\subsection{Notations and geometric preliminaries}

Let $\nu$ be the inner unit normal to $\Sigma$ and $\tau$ be the tangent unit vector field to $\Sigma$ such that the basis $(\tau, \nu)$ is direct in each point of $\Sigma$. Denote by $s$ a curvilinear abscissa (arc length) along $\Sigma$ oriented according to $\tau$. Thus, the scaling is given by a parameterization of the thin shell $\Omega_{-}^{\delta}$ by the manifold $\Omega_{-}=\Sigma \times(0,1)$ through the mapping

$$
\Omega_{-} \longrightarrow \Omega_{-}^{\delta}, \quad(s, z) \longrightarrow s+\delta z \nu(s) .
$$

We identify $\Sigma$ with $\Sigma \times\{0\}$ and we set $\Sigma_{-}=\Sigma \times\{1\}, \Gamma_{-}=\partial \Omega_{-} \backslash\left(\Sigma \cup \Sigma_{-}\right)$and $\Omega=\Omega_{+} \cup \Sigma \cup \Omega_{-}$. Each function $\psi$ defined on $\Omega_{-}^{\delta}$ is associated to a function $\widehat{\psi}$ defined in $\Omega_{-}$through the variable change (3.1) by $\widehat{\psi}(s, z):=\psi(s, y)$. Denoting by $R=R(s)$ the curvature of $\Sigma$ at $s$, using the Frenet's relations $\partial_{s} \nu=-R \tau$ and $\partial_{s} \tau=R \nu$, we obtain

$$
\partial_{z}=\nu_{1} \partial_{x}+\nu_{2} \partial_{y} \quad \text { and } \quad \partial_{s}=(1-R z)\left(\nu_{2} \partial_{x}-\nu_{1} \partial_{y}\right) .
$$

In $\Omega_{-}^{\delta}$, a given vector field $\varphi$ will be decomposed into its normal and tangential components: $\varphi=\varphi_{\tau} \tau+\varphi_{\nu} \nu$. By the scaling (3.1), $\varphi$ is transformed into $\hat{\varphi}$ $(s, z)=\varphi_{\tau}(s, \delta z) \tau+\delta \varphi_{\nu}(s, \delta z) \nu$.

Likewise, we express the integrals involved in (2.8) by :

$$
\int_{\Omega_{-}^{\delta}} \psi d \Omega_{-}^{\delta}=\delta \int_{\Sigma} \int_{0}^{1} \widehat{\psi}(1-R \delta z) d s d z .
$$

\subsection{The formulation in a fixed domain}

After the scaling (3.1), problem (2.8) is transformed into a new problem posed over the set $\Omega$, where now the operators depend on the small parameter $\delta$. Let us denote by $u_{-}^{\delta}, w_{-}^{\delta}, \phi_{-}^{\delta}, \theta_{-}^{\delta}, \varphi_{-}, \psi_{-}, \zeta_{-}$and $\eta_{-}$the associated images, respectively of $u, w, \phi, \theta, \varphi, \psi, \zeta$ and $\eta$, namely,

$$
\begin{aligned}
& u_{-}^{\delta}:=\widehat{u_{\mid \Omega_{-}^{\delta}}}, \quad w_{-}^{\delta}:=\widehat{w_{\mid \Omega_{-}^{\delta}}}, \quad \phi_{-}^{\delta}:=\widehat{\phi_{\mid \Omega_{-}^{\delta}}}, \theta_{-}^{\delta}:=\widehat{\theta_{\mid \Omega_{-}^{\delta}}}, \\
& \varphi_{-}:=\widehat{\varphi}, \quad \psi_{-}:=\widehat{\psi}, \quad \zeta_{-}:=\widehat{\zeta}, \eta_{-}:=\widehat{\eta} .
\end{aligned}
$$

The following functional setting is involved

$$
\begin{aligned}
& W^{\delta}(\Omega)=\left\{\begin{array}{l}
\left(\psi_{+}, \psi_{-}\right) \in H^{2}\left(\Omega_{+}\right) \times H^{2}\left(\Omega_{-}\right) ; \\
\psi_{+\mid \Sigma}=\psi_{-\mid \Sigma} ; \partial_{\nu} \psi_{+\left.\right|_{\Sigma}}=\delta^{-1} \partial_{z} \psi_{-\left.\right|_{\Sigma}} ; \\
\psi_{+\mid \Gamma_{+}}=\left.\partial_{\nu} \psi_{+}\right|_{\Gamma_{+}}=0 \text { and } \psi_{-\mid \Gamma_{-}}=\left.\partial_{\nu} \psi_{-}\right|_{\Gamma_{-}}=0
\end{array}\right\}, \\
& V^{\delta}(\Omega)=\left\{\left(\psi_{+}, \psi_{-}\right) \in H^{1}\left(\Omega_{+}\right) \times H^{1}\left(\Omega_{-}\right) ; \psi_{-\mid \Sigma}=\psi_{+\mid \Sigma}, \psi_{+\mid \Gamma_{+}}=0 ;\right. \\
& \left.\psi_{-\mid \Gamma_{-}}=0\right\}, \\
& U^{\delta}(\Omega)=\left\{\begin{array}{l}
\left(\varphi_{+}, \varphi_{-}\right) \in H^{1}\left(\Omega_{+}\right) \times H^{1}\left(\Omega_{-}\right) ; \varphi_{-\tau \mid \Sigma}=\varphi_{+\tau} \mid \Sigma \\
\varphi_{-\nu} \mid \Sigma=\delta \varphi_{+_{\nu} \mid \Sigma} ; \varphi_{+\mid \Gamma_{+}}=0 ; \varphi_{-\mid \Gamma_{-}}=0
\end{array}\right\} .
\end{aligned}
$$


Let $\psi \in W^{\delta}(\Omega), \varphi \in U^{\delta}(\Omega)$. We set

$$
\begin{aligned}
& \gamma_{T}^{\delta}(\psi)=(1-R \delta z)^{-1} \partial_{s}\left((1-R \delta z)^{-1} \partial_{s} \psi\right)-R(1-R \delta z)^{-1} \partial_{z} \psi, \\
& \gamma_{S}^{\delta}(\psi)=-\partial_{z}\left((1-R \delta z)^{-1} \partial_{s} \psi\right), \quad \gamma_{N}^{\delta}(\psi)=\partial_{z}^{2} \psi, \\
& \varepsilon_{T}^{\delta}(\varphi)=(1-R \delta z)^{-1} \partial_{s} \varphi_{\tau}-\delta^{-1} R(1-R \delta z)^{-1} \varphi_{\nu}, \quad \varepsilon_{N}^{\delta}(\varphi)=\partial_{z} \varphi_{\nu}, \\
& \varepsilon_{S}^{\delta}(\varphi)=\frac{1}{2} \delta\left(R(1-R \delta z)^{-1} \varphi_{\tau}+\delta^{-1}(1-R \delta z)^{-1} \partial_{s} \varphi_{\nu}+\delta^{-1} \partial_{z} \varphi_{\tau}\right),
\end{aligned}
$$

and to shorten the notation we denote

$$
\begin{aligned}
& N_{T}^{\delta}\left(u_{-}^{\delta}, w_{-}^{\delta}\right)=\varepsilon_{T}^{\delta}\left(u_{-}^{\delta}\right)+0.5(1-R \delta z)^{-2}\left(\partial_{s} w_{-}^{\delta}\right)^{2} \\
& N_{S}^{\delta}\left(u_{-}^{\delta}, w_{-}^{\delta}\right)=2 \varepsilon_{S}^{\delta}\left(u_{-}^{\delta}\right)-(1-R \delta z)^{-1} \partial_{s} w_{-}^{\delta} \partial_{z} w_{-}^{\delta}, \\
& N_{N}^{\delta}\left(u_{-}^{\delta}, w_{-}^{\delta}\right)=\varepsilon_{N}^{\delta}\left(u_{-}^{\delta}\right)+0.5\left(\partial_{z} w_{-}^{\delta}\right)^{2} .
\end{aligned}
$$

Using the above notations and formulas of change of variables and denoting by $u^{\delta}:=\left(u_{+}^{\delta}, u_{-}^{\delta}\right), w^{\delta}:=\left(w_{+}^{\delta}, w_{-}^{\delta}\right), \phi^{\delta}:=\left(\phi_{+}^{\delta}, \phi_{-}^{\delta}\right)$ and $\theta^{\delta}:=\left(\theta_{+}^{\delta}, \theta_{-}^{\delta}\right)$ where $u_{+}^{\delta}=u_{\mid \Omega_{+}}, w_{+}^{\delta}=w_{\mid \Omega_{+}}, \phi_{+}^{\delta}=\phi_{\mid \Omega_{+}}, \theta_{+}^{\delta}=\theta_{\mid \Omega_{+}}$, we see that $\left(u^{\delta}, w^{\delta}, \phi^{\delta}, \theta^{\delta}\right)$ is solution of the following variational problem:

$$
\begin{gathered}
u^{\delta} \in L^{\infty}\left(0, T ; U^{\delta}(\Omega)\right), \quad\left(u^{\delta}\right)^{\prime} \in L^{\infty}\left(0, T ;\left(L^{2}(\Omega)\right)^{2}\right), \\
w^{\delta} \in L^{\infty}\left(0, T ; W^{\delta}(\Omega)\right),\left(w^{\delta}\right)^{\prime} \in L^{\infty}\left(0, T ; V^{\delta}(\Omega)\right), \\
\phi^{\delta}, \theta^{\delta} \in L^{\infty}\left(0, T ; L^{2}(\Omega)\right) \cap L^{2}\left(0, T ; V^{\delta}(\Omega)\right) \\
\rho_{+}\left\langle\left(u_{+}^{\delta}\right)^{\prime}, \varphi_{+}\right\rangle_{\Omega_{+}}^{\prime}+\rho_{-}\left\langle\left(u_{-}^{\delta}\right)^{\prime}, \varphi_{-}\right\rangle_{\Omega_{-}}^{\prime}+\rho_{+}\left\langle\left(w_{+}^{\delta}\right)^{\prime}, \psi_{+}\right\rangle_{\Omega_{+}}^{\prime}+\rho_{-}\left\langle\left(w_{-}^{\delta}\right)^{\prime}, \psi_{-}\right\rangle_{\Omega_{-}}^{\prime} \\
+\rho_{+} b_{+}\left(\left(w_{+}^{\delta}\right)^{\prime}, \psi_{+}\right)^{\prime}+\rho_{-} b_{-}^{\delta}\left(\left(w_{-}^{\delta}\right)^{\prime}, \psi_{-}\right)^{\prime}+a_{+}\left(w_{+}^{\delta}, \psi_{+}\right)+a_{-}^{\delta}\left(w_{-}^{\delta}, \psi_{-}\right) \\
+\lambda_{+} c_{+}\left(\phi_{+}^{\delta}, \varphi_{+}\right)+\lambda_{-} c_{-}^{\delta}\left(\phi_{-}^{\delta}, \varphi_{-}\right)-\lambda_{+} b_{+}\left(\theta_{+}^{\delta}, \psi_{+}\right)-\lambda_{-} b_{-}^{\delta}\left(\theta_{-}^{\delta}, \psi_{-}\right) \\
+\rho_{+}\left\langle\phi_{+}^{\delta}, \zeta_{+}\right\rangle_{\Omega_{+}}^{\prime}+\rho_{-}\left\langle\phi_{-}^{\delta}, \zeta_{-}\right\rangle_{\Omega_{-}}^{\prime}+k_{+} b_{+}\left(\phi_{+}^{\delta}, \zeta_{+}\right)+k_{-} b_{-}^{\delta}\left(\phi_{-}^{\delta}, \zeta_{-}\right) \\
-\lambda_{+} d_{+}\left(u_{+}^{\prime}, \zeta_{+}\right)-\lambda_{-} d_{-}^{\delta}\left(\left(u_{-}^{\delta}\right)^{\prime}, \zeta_{-}\right)+\rho_{+}\left\langle\theta_{+}, \eta_{+}\right\rangle_{\Omega_{+}}^{\prime}+\rho_{-}\left\langle\theta_{-}^{\delta}, \eta_{-}\right\rangle_{\Omega_{-}}^{\prime} \\
+\lambda_{+} b_{+}\left(\left(w_{+}^{\delta}\right)^{\prime}, \eta_{+}\right)+\lambda_{-} b_{-}^{\delta}\left(\left(w_{-}^{\delta}\right)^{\prime}, \eta_{-}\right)+k_{+} b_{+}\left(\theta_{+}^{\delta}, \eta_{+}\right)+k_{-} b_{-}^{\delta}\left(\theta_{-}^{\delta}, \eta_{-}\right) \\
+N_{+}\left(u_{+}^{\delta}, w_{+}^{\delta}, \varphi_{+}, \psi_{+}\right)+N_{-}^{\delta}\left(u_{-}^{\delta}, w_{-}^{\delta}, \varphi_{-}, \psi_{-}\right)=0
\end{gathered}
$$

$\forall(\varphi, \psi, \zeta, \eta) \in U^{\delta}(\Omega) \times W^{\delta}(\Omega) \times V^{\delta}(\Omega) \times V^{\delta}(\Omega)$, with initial conditions

$$
\begin{aligned}
& u^{\delta}(0)=u_{0}^{\delta}, \quad\left(u^{\delta}\right)^{\prime}(0)=u_{1}^{\delta}, \quad w^{\delta}(0)=w_{0}^{\delta}, \\
& \left(w^{\delta}\right)^{\prime}(0)=w_{1}^{\delta}, \quad \theta^{\delta}(0)=\theta_{0}^{\delta}, \quad \phi^{\delta}(0)=\phi_{0}^{\delta} \quad \text { in } \Omega,
\end{aligned}
$$

where

$$
\begin{aligned}
& a_{+}\left(w_{+}^{\delta}, \psi_{+}\right)=D_{+} \int_{\Omega_{+}}\left\{\left(\partial_{x}^{2} w_{+}^{\delta}+\mu_{+} \partial_{y}^{2} w_{+}^{\delta}\right) \partial_{x}^{2} \psi_{+}+2\left(1-\mu_{+}\right) \partial_{x y}^{2} w_{+}^{\delta} \partial_{x y}^{2} \psi_{+}\right. \\
& \left.\quad+\left(\partial_{y}^{2} w_{+}^{\delta}+\mu_{+} \partial_{x}^{2} w_{+}^{\delta}\right) \partial_{y}^{2} \psi_{+}\right\} d \Omega_{+}
\end{aligned}
$$




$$
\begin{aligned}
& b_{+}\left(w_{+}^{\delta}, \psi_{+}\right)=\int_{\Omega_{+}} \nabla w_{+}^{\delta} \nabla \psi_{+} d \Omega_{+}, \quad c_{+}\left(\phi_{+}^{\delta}, \varphi_{+}\right)=\int_{\Omega_{+}} \nabla \phi_{+}^{\delta} \varphi_{+} d \Omega_{+}, \\
& d_{+}\left(u_{+}^{\prime}, \zeta_{+}\right)=\int_{\Omega_{+}} u_{+}^{\prime} \nabla \zeta_{+} d \Omega_{+}, a_{-}^{\delta}\left(w_{-}^{\delta}, \psi_{-}\right)=D_{-} \int_{\Omega_{-}}\left\{\left(\gamma_{T}^{\delta}\left(w_{-}^{\delta}\right)\right.\right. \\
& \left.+\delta^{-2} \mu_{-} \gamma_{N}^{\delta}\left(w_{-}^{\delta}\right)\right) \gamma_{T}^{\delta}\left(\psi_{-}^{\delta}\right)+2 \delta^{-2}\left(1-\mu_{-}\right) \gamma_{S}^{\delta}\left(w_{-}^{\delta}\right) \gamma_{S}^{\delta}\left(\psi_{-}^{\delta}\right) \\
& \left.+\delta^{-2}\left(\delta^{-2} \gamma_{N}^{\delta}\left(w_{-}^{\delta}\right)+\mu_{-} \gamma_{T}^{\delta}\left(w_{-}^{\delta}\right)\right) \gamma_{N}^{\delta}\left(\psi_{-}\right)\right\}(1-R \delta z) d s d z \\
& b_{-}^{\delta}\left(w_{-}^{\delta}, \psi_{-}\right)=\int_{0}^{1} \int_{\Sigma}\left\{(1-R \delta z)^{-2} \partial_{s} w_{-}^{\delta} \partial_{s} \psi_{-}+\delta^{-2} \partial_{z} w_{-}^{\delta} \partial_{z} \psi_{-}\right\}(1-R \delta z) d s d z \text {, } \\
& c_{-}^{\delta}\left(\phi_{-}^{\delta}, \varphi_{-}\right)=\int_{0}^{1} \int_{\Sigma}\left(-(1-R \delta z)^{-1} \partial_{s} \phi_{-}^{\delta} \varphi_{-\tau}+\delta^{-2} \partial_{z} \phi_{-}^{\delta} \varphi_{-\nu}\right)(1-R \delta z) d s d z, \\
& d_{-}^{\delta}\left(\left(u_{-}^{\delta}\right)^{\prime}, \zeta_{-}\right)=\int_{0}^{1} \int_{\Sigma}\left(-(1-R \delta z)^{-1}\left(u_{-\tau}^{\delta}\right)^{\prime} \partial_{s} \zeta+\delta^{-2}\left(u_{-\nu}^{\delta}\right)^{\prime} \partial_{z} \zeta\right)(1-R \delta z) d s d z, \\
& N_{+}\left(u_{+}^{\delta}, w_{+}^{\delta}, \varphi_{+}, \psi_{+}\right)=\left\langle C\left[\epsilon\left(u_{+}^{\delta}\right)+f\left(\nabla w_{+}^{\delta}\right)\right], \epsilon\left(\varphi_{+}\right)\right\rangle_{\Omega_{+}} \\
& +\left\langle C\left[\epsilon\left(u_{+}^{\delta}\right)+f\left(\nabla w_{+}^{\delta}\right)\right] \nabla w_{+}^{\delta}, \nabla \psi_{+}\right\rangle_{\Omega_{+}}, N_{-}^{\delta}\left(u_{-}^{\delta}, w_{-}^{\delta}, \varphi_{-}, \psi_{-}\right) \\
& =\frac{E_{-}}{1-\mu_{-}^{2}} \int_{\Omega_{-}}\left\{\left[N_{T}^{\delta}\left(u_{-}^{\delta}, w_{-}^{\delta}\right)+\delta^{-2} \mu_{-} N_{N}^{\delta}\left(u_{-}^{\delta}, w_{-}^{\delta}\right)\right]\right. \\
& \times\left[\varepsilon_{T}^{\delta}\left(\varphi_{-}\right)+(1-R \delta z)^{-2} \partial_{s} w_{-}^{\delta} \partial_{s} \psi_{-}\right]+\frac{\left(1-\mu_{-}\right)}{2} \delta^{-2} N_{S}^{\delta}\left(u_{-}^{\delta}, w_{-}^{\delta}\right) \\
& \times\left[2 \varepsilon_{S}^{\delta}\left(\varphi_{-}\right)-(1-R \delta z)^{-1}\left(\partial_{s} w_{-}^{\delta} \partial_{z} \psi_{-}+\partial_{z} w_{-}^{\delta} \partial_{s} \psi_{-}\right)\right]+\frac{1}{\delta^{2}}\left[\frac{1}{\delta^{2}} N_{N}^{\delta}\left(u_{-}^{\delta}, w_{-}^{\delta}\right)\right. \\
& \left.\left.+\mu_{-} N_{T}^{\delta}\left(u_{-}^{\delta}, w_{-}^{\delta}\right)\right]\left[\varepsilon_{N}^{\delta}\left(\varphi_{-}\right)+\partial_{z} w_{-}^{\delta} \partial_{z} \psi_{-}\right]\right\}(1-R \delta z) d s d z .
\end{aligned}
$$

\section{Ventcel's Model}

\subsection{A priori estimates}

The first step in order to obtain the limit model consists on the establishment of a priori bounds which allow us to pass to the limit in the scaled variational problem. Let $\left(u^{\delta}, w^{\delta}, \phi^{\delta}, \theta^{\delta}\right)$ be a solution of the problem (3.2). Denote

$$
\begin{aligned}
& E^{\delta}(t)=\frac{1}{2}\left\{\rho_{+}\left\|\left(u_{+}^{\delta}\right)^{\prime}(t)\right\|_{L^{2}\left(\Omega_{+}\right)}^{2}+\rho_{+}\left\|\left(w_{+}^{\delta}\right)^{\prime}(t)\right\|_{L^{2}\left(\Omega_{+}\right)}^{2}+\rho_{+}\left\|\theta_{+}^{\delta}(t)\right\|_{L^{2}\left(\Omega_{+}\right)}^{2}\right. \\
& \quad+\rho_{+}\left\|\phi_{+}^{\delta}(t)\right\|_{L^{2}\left(\Omega_{+}\right)}^{2}+\rho_{+} b_{+}\left(\left(w_{+}^{\delta}\right)^{\prime}(t),\left(w_{+}^{\delta}\right)^{\prime}(t)\right)+a_{+}\left(w_{+}^{\delta}(t), w_{+}^{\delta}(t)\right) \\
& \quad+N_{+}\left(u_{+}^{\delta}(t), w_{+}^{\delta}(t), u_{+}^{\delta}(t), 1 / 2 w_{+}^{\delta}(t)\right)+\rho_{-}\left\|\theta_{-}^{\delta}(t)\right\|_{L^{2}\left(\Omega_{-}\right)}^{2} \\
& \quad+\rho_{-}\left\|\phi_{-}^{\delta}(t)\right\|_{L^{2}\left(\Omega_{-}\right)}^{2}+\rho_{-}\left\|\left(u_{-}^{\delta}\right)^{\prime}(t)\right\|_{L^{2}\left(\Omega_{-}\right)}^{2}+\rho_{-}\left\|\left(w_{-}^{\delta}\right)^{\prime}(t)\right\|_{L^{2}\left(\Omega_{-}\right)}^{2}
\end{aligned}
$$




$$
\begin{aligned}
& +\rho_{-} b_{-}^{\delta}\left(\left(w_{-}^{\delta}\right)^{\prime}(t),\left(w_{-}^{\delta}\right)^{\prime}(t)\right)+a_{-}^{\delta}\left(w_{-}^{\delta}(t), w_{-}^{\delta}(t)\right) \\
& \left.+N_{-}^{\delta}\left(u_{-}^{\delta}(t), w_{-}^{\delta}(t), u_{-}^{\delta}(t), 1 / 2 w_{-}^{\delta}(t)\right)\right\} .
\end{aligned}
$$

Theorem 1. Suppose that $E^{\delta}(0)$ is bounded independently of $\delta$. Then: a) $w^{\delta}$ is bounded independently of $\delta$ in $L^{\infty}\left(0, T ; W^{\delta}(\Omega)\right) ; \quad$ b) $\left(w^{\delta}\right)^{\prime}$ is bounded independently of $\delta$ in $L^{\infty}\left(0, T ; V^{\delta}(\Omega)\right) ; \quad$ c) $u^{\delta}$ is bounded independently of $\delta$ in $\left.L^{\infty}\left(0, T ; U^{\delta}(\Omega)\right) ; d\right)\left(u^{\delta}\right)^{\prime}$ is bounded independently of $\delta$ in $L^{\infty}\left(0, T ;\left(L^{2}(\Omega)\right)^{2}\right)$; e) $\phi^{\delta}$ and $\theta^{\delta}$ are bounded independently of $\delta$ in $\left.L^{\infty}\left(0, T ; L^{2}(\Omega)\right) ; f\right) \frac{1}{\delta}\left(\partial_{z} w^{\delta}\right)^{\prime}$, $\gamma_{T}^{\delta}\left(w_{-}^{\delta}\right), \frac{1}{\delta} \gamma_{S}^{\delta}\left(w_{-}^{\delta}\right), \frac{1}{\delta^{2}} \gamma_{N}^{\delta}\left(w_{-}^{\delta}\right), N_{T}^{\delta}\left(u_{-}^{\delta}, w_{-}^{\delta}\right), \frac{1}{\delta^{2}} N_{N}^{\delta}\left(u_{-}^{\delta}, w_{-}^{\delta}\right)$ and $\frac{1}{\delta} N_{S}^{\delta}\left(u_{-}^{\delta}, w_{-}^{\delta}\right)$ are bounded independently of $\delta$ in $L^{\infty}\left(0, T ;\left(L^{2}\left(\Omega_{-}\right)\right), \quad g\right) \nabla \phi_{+}^{\delta}$ and $\nabla \theta_{+}^{\delta}$ are bounded independently of $\delta$ in $\left.L^{2}\left(0, T ; L^{2}\left(\Omega_{+}\right)\right), h\right) \frac{1}{\delta} \partial_{z} \phi_{-}^{\delta}, \frac{1}{\delta} \partial_{z} \theta_{-}^{\delta}, \partial_{s} \phi_{-}^{\delta^{+}}$and $\partial_{s} \theta_{-}^{\delta}$ are bounded independently of $\delta$ in $L^{2}\left(0, T ; L^{2}\left(\Omega_{-}\right)\right)$.

Proof. The proof is standard and follows by classical energy type argument. Indeed, taking $\varphi=\left(u^{\delta}\right)^{\prime}, \psi=\left(w^{\delta}\right)^{\prime}, \eta=\theta^{\delta}$ and $\zeta=\phi^{\delta}$ in the variational formulation (3.2), integrating from 0 to $t$, we get

$$
\begin{aligned}
& E^{\delta}(t)+k_{+} \int_{0}^{t}\left\|\nabla \theta_{+}^{\delta}(t)\right\|_{L^{2}\left(\Omega_{+}\right)}^{2} d t+k_{+} \int_{0}^{t}\left\|\nabla \phi_{+}^{\delta}(t)\right\|_{L^{2}\left(\Omega_{+}\right)}^{2} d t \\
& \quad+k_{-} \int_{0}^{t} b_{-}^{\delta}\left(\theta_{-}^{\delta}, \theta_{-}^{\delta}\right) d t+k_{-} \int_{0}^{t} b_{-}^{\delta}\left(\phi_{-}^{\delta}, \phi_{-}^{\delta}\right) d t=E^{\delta}(0),
\end{aligned}
$$

first for smooth solutions which is then extended by density to all weak solutions. Moreover, it is clear that there exists two positive constants $\delta_{0}$ and $c$ such that

$$
c^{-1} \leq\left\|(1-R \delta z)^{-1}\right\|_{L^{\infty}(\Omega)}+\|1-R \delta z\|_{L^{\infty}(\Omega)} \leq c, \quad \forall \delta, \quad 0<\delta \leq \delta_{0} .
$$

Using the Poincare and Korn inequalities, we get the previous a priori bounds.

The above a priori bounds allow us to extract convergent subsequences (still indexed by $\delta$ for notational convenience) such that $w^{\delta} \rightarrow \widetilde{w}$ weakly* in $L^{\infty}\left(0, T ; H^{2}(\Omega)\right),\left(w^{\delta}\right)^{\prime} \rightarrow \widetilde{w}^{\prime}$ weakly* in $L^{\infty}\left(0, T ; H^{1}(\Omega)\right)$, $u^{\delta} \rightarrow \widetilde{u}$ weakly*in $L^{\infty}\left(0, T ;\left(H^{1}(\Omega)\right)^{2}\right),\left(u^{\delta}\right)^{\prime} \rightarrow \widetilde{u}^{\prime}$ weakly* in $L^{\infty}\left(0, T ;\left(L^{2}(\Omega)\right)^{2}\right)$, $\theta^{\delta} \rightarrow \tilde{\theta}$ weakly* in $L^{\infty}\left(0, T ;\left(L^{2}(\Omega)\right)\right.$ and weakly in $L^{2}\left(0, T ; H^{1}(\Omega)\right)$, $\phi^{\delta} \rightarrow \tilde{\phi}$ weakly* in $L^{\infty}\left(0, T ;\left(L^{2}(\Omega)\right)\right)$ and weakly in $L^{2}\left(0, T ; H^{1}(\Omega)\right)$.

Setting

$W\left(\Omega_{+}\right)=\left\{w \in H^{2}\left(\Omega_{+}\right) ; w_{\mid \Gamma_{+}}=\left.\partial_{\nu} w\right|_{\Gamma_{+}}=0, w_{\mid \Sigma} \in H_{0}^{2}(\Sigma), \partial_{\nu} w \in H_{0}^{1}(\Sigma)\right\}$, $V\left(\Omega_{+}\right)=\left\{w \in H^{1}\left(\Omega_{+}\right) ; w_{\mid \Gamma_{+}}=0, w_{\mid \Sigma} \in H_{0}^{1}(\Sigma)\right\}$, $U\left(\Omega_{+}\right)=\left\{u \in\left(H^{1}\left(\Omega_{+}\right)\right)^{2} ; u_{\mid \Gamma_{+}}=0, u_{\tau} \in H_{0}^{1}(\Sigma)\right\}$,

we have the following result: 
Theorem 2. Denote by $\widetilde{u}:=\left(\widetilde{u}_{+}, \widetilde{u}_{-}\right), \widetilde{w}:=\left(\widetilde{w}_{+}, \widetilde{w}_{-}\right), \widetilde{\phi}:=\left(\widetilde{\phi}_{+}, \widetilde{\phi}_{-}\right)$and $\widetilde{\theta}:=\left(\widetilde{\theta}_{+}, \widetilde{\theta}_{-}\right)$, where $\widetilde{w}_{ \pm}=\left.\widetilde{w}\right|_{\Omega_{ \pm}}, \widetilde{u}_{ \pm}=\left.\widetilde{u}\right|_{\Omega_{ \pm}}, \widetilde{\phi}_{ \pm}=\left.\widetilde{\phi}\right|_{\Omega_{ \pm}}$and $\widetilde{\theta}_{ \pm}=\left.\widetilde{\theta}\right|_{\Omega_{ \pm}}$, we see that the limit $(\widetilde{u}, \widetilde{w}, \widetilde{\phi}, \widetilde{\theta})$ is characterized as follows:

$$
\begin{aligned}
& \widetilde{w}_{+} \in L^{\infty}\left(0, T ; W\left(\Omega_{+}\right)\right), \quad\left(\widetilde{w}_{+}\right)^{\prime} \in L^{\infty}\left(0, T ; V\left(\Omega_{+}\right)\right), \\
& \left(\partial_{\nu} \widetilde{w}_{+}\right)^{\prime} \in L^{\infty}\left(0, T ;\left(L^{2}(\Sigma)\right), \quad \widetilde{u}_{+} \in L^{\infty}\left(0, T ; U\left(\Omega_{+}\right)\right),\right. \\
& \left(\widetilde{u}_{+}\right)^{\prime} \in L^{\infty}\left(0, T ;\left(L^{2}\left(\Omega_{+}\right)\right)^{2}\right), \quad\left(\widetilde{u}_{+}\right)_{\left.\right|_{\Sigma}}^{\prime} \in L^{\infty}\left(0, T ;\left(\left(L^{2}(\Sigma)\right)^{2}\right),\right. \\
& \widetilde{\theta}_{+}, \widetilde{\phi}_{+} \in L^{\infty}\left(0, T ;\left(L^{2}\left(\Omega_{+}\right)\right) \cap L^{2}\left(0, T ; V\left(\Omega_{+}\right)\right) .\right.
\end{aligned}
$$

Moreover, we have

$$
\widetilde{w}_{-}=\widetilde{w}_{+\mid \Sigma}, \quad \widetilde{\phi}_{-}=\widetilde{\phi}_{+\mid \Sigma}, \quad \widetilde{\theta}_{-}=\widetilde{\theta}_{+\mid \Sigma}, \quad \widetilde{u}_{-\tau}=\left.\widetilde{u}_{+\tau}\right|_{\Sigma}, \quad \widetilde{u}_{-\nu}=0 .
$$

Proof. We refer to the proof of Proposition 2 in [10]. Indeed, the same arguments as in [10] apply to $\left(u^{\delta}, w^{\delta}\right)$. As for $\left(\phi^{\delta}, \theta^{\delta}\right)$, we can easily show that $\widetilde{\phi}_{-}=\widetilde{\phi}_{+\mid \Sigma}, \widetilde{\theta}_{-}=\widetilde{\theta}_{+\mid \Sigma}$.

Remark 3. Hereafter, for the sake of simplicity, we do not write the traces on $\Sigma$ of a function defined on $\Omega_{+}$by an explicit notation since the latter will be always clear from the context.

Theorem 3. The following weak convergences hold true
a) $\int_{0}^{1} \gamma_{T}^{\delta}\left(w_{-}^{\delta}\right)(1-R \delta z) d z \rightarrow \gamma_{T}\left(\widetilde{w}_{+}\right)$weakly* in $L^{\infty}\left(0, T ; L^{2}(\Sigma)\right)$
b) $\int_{0}^{1} \frac{1}{\delta} \gamma_{S}^{\delta}\left(w_{-}^{\delta}\right)(1-R \delta z) d z \rightarrow \gamma_{S}\left(\widetilde{w}_{+}\right)$weakly* in $L^{\infty}\left(0, T ; L^{2}(\Sigma)\right)$,
c) $\int_{0}^{1} \frac{1}{\delta^{2}} \gamma_{N}^{\delta}\left(w_{-}^{\epsilon}\right)(1-R \delta z) d z \rightarrow-\mu_{-} \gamma_{T}\left(\widetilde{w}_{+}\right)$weakly* in $L^{\infty}\left(0, T ; L^{2}(\Sigma)\right)$,
d) $\int_{0}^{1} N_{T}^{\delta}\left(u^{\delta}, w^{\delta}\right)(1-R \delta z) d z \rightarrow N_{T}\left(\widetilde{u}_{+}, \widetilde{w}_{+}\right)$weakly* in $L^{\infty}\left(0, T ; L^{2}(\Sigma)\right)$,
e) $\int_{0}^{1} \frac{1}{\delta^{2}} N_{N}^{\delta}\left(u^{\delta}, w^{\delta}\right)(1-R \delta z) d z \rightarrow-\mu_{-} N_{T}\left(\widetilde{u}_{+}, \widetilde{w}_{+}\right)$weakly* in $L^{\infty}\left(0, T ; L^{2}(\Sigma)\right)$,
f) $\int_{0}^{1} \frac{1}{\delta} N_{S}^{\delta}\left(u^{\delta}, w^{\delta}\right)(1-R \delta z) d z \rightarrow 0$ weakly* in $L^{\infty}\left(0, T ; L^{2}(\Sigma)\right)$,
g) $\int_{0}^{1} \frac{1}{\delta} \partial_{z} \phi_{-}^{\delta}(1-R \delta z) d z \rightarrow \frac{\lambda_{-}}{k_{-}}\left(\widetilde{u}_{+\nu}\right)^{\prime}$ weakly in $L^{2}\left(0, T ; L^{2}(\Sigma)\right)$, 
$h) \int_{0}^{1} \frac{1}{\delta} \partial_{z} \theta_{-}^{\delta}(1-R \delta z) d z \rightarrow \frac{\lambda_{-}}{k_{-}}\left(\partial_{\nu} \widetilde{w}_{+}\right)^{\prime}$ weakly in $L^{2}\left(0, T ; L^{2}(\Sigma)\right)$,

where

$$
\begin{aligned}
& N_{T}\left(\widetilde{u}_{+}, \widetilde{w}_{+}\right)=\partial_{s} \widetilde{u}_{+\tau}-R(s) \widetilde{u}_{+\nu}+\frac{1}{2}\left(\partial_{s} \widetilde{w}_{+}\right)^{2}, \gamma_{T}\left(\widetilde{w}_{+}\right)=\partial_{s}^{2} \widetilde{w}_{+}-R(s) \partial_{\nu} \widetilde{w}_{+}, \\
& \gamma_{S}\left(\widetilde{w}_{+}\right)=-\partial_{s} \partial_{\nu} \widetilde{w}_{+}-R(s) \partial_{s} \widetilde{w}_{+} .
\end{aligned}
$$

Proof. The first six limits are established as in the proof of Proposition 3 in [10] with $\eta_{ \pm}=0, \zeta_{ \pm}=0$ in the variational formulation (3.2). The proof of the other limits can be achieved by using the same arguments. Indeed, we set $\psi_{+}=0, \psi_{-}=0, \varphi_{+}=0, \varphi_{-}=0, \eta_{+}=0, \eta_{-}=0, \zeta_{+}=0, \zeta_{-}=\delta \kappa(s) z$ in (3.2), where $\kappa$ is a smooth enough function that doesn't depend on $z$. This yields

$$
\int_{\Sigma} \int_{0}^{1}\left(\frac{k_{-}}{\delta} \partial_{z} \phi_{-}^{\delta}-\frac{\lambda_{-}}{\delta}\left(u_{-\nu}^{\delta}\right)^{\prime}\right)(1-R \delta z) d z d s \rightarrow 0 \quad \text { in } D^{\prime}(0, T) .
$$

We deduce that $\int_{0}^{1}\left(\frac{k_{-}}{\delta} \partial_{z} \phi_{-}^{\delta}-\frac{\lambda_{-}}{\delta}\left(u_{-\nu}^{\delta}\right)^{\prime}\right)(1-R \delta z) d z$ converges to 0 weakly in $L^{2}\left(0, T ; L^{2}(\Sigma)\right)$. Owing to the fact that $\int_{0}^{1} \frac{1}{\delta}\left(u_{-\nu}^{\delta}\right)^{\prime} d z \rightarrow\left(\widetilde{u}_{+\nu}\right)_{\left.\right|_{\Sigma}}^{\prime}$ weakly in $L^{2}\left(0, T ; L^{2}(\Sigma)\right)$, we conclude that $\int_{0}^{1} \frac{1}{\delta} \partial_{z} \phi_{-}^{\delta}(1-R \delta z) d z \rightarrow \frac{\lambda_{-}}{k_{-}}\left(\widetilde{u}_{+\nu}\right)^{\prime}$ weakly in $L^{2}\left(0, T ; L^{2}(\Sigma)\right)$.

Similarly, we get the last convergence by applying (3.2) with the test functions $\psi_{+}=0, \psi_{-}=0, \varphi_{+}=0, \varphi_{-}=0, \eta_{+}=0, \eta_{-}=\delta \kappa(s) z, \zeta_{+}=0, \zeta_{-}=0$, and using the fact that $\frac{1}{\delta} \int_{0}^{1}\left(\partial_{z} w_{-}^{\delta}\right)^{\prime} d z \longrightarrow\left(\partial_{\nu} \tilde{w}_{+}\right)^{\prime}$ weakly in $L^{2}\left(0, T ; L^{2}(\Sigma)\right)$.

We can now go to the limit on the problem (3.2). We shall show that the limit functions $\widetilde{u}, \tilde{w}, \tilde{\phi}$ and $\tilde{\theta}$ satisfy a Cauchy-Ventcel problem.

Theorem 4. Suppose that

$$
\begin{aligned}
& \text { a) }\left(w_{+}^{0 \delta}, \int_{0}^{1} w_{-}^{0 \delta} d z\right) \rightarrow\left(w_{+}^{*},\left.w_{+}^{*}\right|_{\Sigma}\right) \quad \text { in } H^{2}\left(\Omega_{+}\right) \times H^{2}(\Sigma), \\
& \text { b) }\left(w_{+}^{1 \delta}, \int_{0}^{1} w_{-}^{1 \delta} d z\right) \rightarrow\left(w_{+}^{* *},\left.w_{+}^{* *}\right|_{\Sigma}\right) \text { in } H^{1}\left(\Omega_{+}\right) \times H^{1}(\Sigma) \text {, } \\
& \delta^{-1} \int_{0}^{1} \partial_{z} w_{-}^{1 \delta} d z \rightarrow w^{* * *} \quad \text { in } L^{2}(\Sigma), \\
& \text { c) }\left(\phi_{+}^{0 \delta}, \int_{0}^{1} \phi_{-}^{0 \delta} d z\right) \rightarrow\left(\phi_{+}^{*},\left.\phi_{+}^{*}\right|_{\Sigma}\right) \quad \text { in } H^{1}\left(\Omega_{+}\right) \times H^{1}(\Sigma) \text {, }
\end{aligned}
$$


d) $\left(\theta_{+}^{0 \delta}, \int_{0}^{1} \theta_{-}^{0 \delta} d z\right) \rightarrow\left(\theta_{+}^{*},\left.\theta_{+}^{*}\right|_{\Sigma}\right) \quad$ in $H^{1}\left(\Omega_{+}\right) \times H^{1}(\Sigma)$,

e) $\left(u_{+}^{0 \delta}, \int_{0}^{1} u_{-\tau}^{0 \delta} d z, \int_{0}^{1} u_{-\nu}^{0 \delta} d z\right) \rightarrow\left(u_{+}^{*},\left.u_{+\tau}^{*}\right|_{\Sigma}, 0\right) \quad$ in $\left[H^{1}\left(\Omega_{+}\right)\right]^{2} \times\left[H^{1}(\Sigma)\right]^{2}$, f) $\left(u_{+}^{1 \delta}, \int_{0}^{1} u_{-\tau}^{1 \delta} d z, \int_{0}^{1} \frac{1}{\delta} u_{-\nu}^{1 \delta} d z\right) \rightarrow\left(u_{+}^{* *}, u^{* * *}\right) \quad$ in $\left[L^{2}\left(\Omega_{+}\right)\right]^{2} \times\left[L^{2}(\Sigma .)\right]^{2}$.

Then, the subsequence $u_{+}^{\delta}$ (respectively $\left.w_{+}^{\delta}, \phi_{+}^{\delta}, \theta_{+}^{\delta},\right)$ converges weakly ${ }^{*}$ in $L^{\infty}(0, T ; U(\Omega))$ (respectively in $L^{\infty}\left(0, T ; W\left(\Omega_{+}\right)\right), L^{\infty}\left(0, T ; V\left(\Omega_{+}\right)\right)$) to $\widetilde{u}_{+}$ (respectively to $\widetilde{w}_{+}, \widetilde{\phi}_{+}, \widetilde{\theta}_{+}$) which satisfy the Cauchy-Ventcel problem

$$
\begin{aligned}
& \widetilde{w}_{+} \in L^{\infty}\left(0, T ; W\left(\Omega_{+}\right)\right),\left(\widetilde{w}_{+}\right)^{\prime} \in L^{\infty}\left(0, T ; V\left(\Omega_{+}\right)\right), \\
& \left(\partial_{\nu} \widetilde{w}_{+}\right)^{\prime} \in L^{\infty}\left(0, T ; L^{2}(\Sigma)\right), \widetilde{u}_{+} \in L^{\infty}\left(0, T ; U\left(\Omega_{+}\right)\right), \\
& \left(\widetilde{u}_{+}\right)^{\prime} \in L^{\infty}\left(0, T ;\left[L^{2}\left(\Omega_{+}\right)\right]^{2}\right),\left(\widetilde{u}_{+\mid \Sigma}\right)^{\prime} \in L^{\infty}\left(0, T ;\left(L^{2}(\Sigma)\right)^{2}\right), \\
& \tilde{\theta}_{+}, \tilde{\phi}_{+} \in L^{\infty}\left(0, T ; L^{2}\left(\Omega_{+}\right)\right) \cap L^{2}\left(0, T ; V\left(\Omega_{+}\right)\right), \\
\left\{\rho_{+}\right. & \left\langle\widetilde{u}_{+}^{\prime}, \varphi\right\rangle_{\Omega+}^{\prime}+\rho_{-}\left\langle\widetilde{u}_{+}^{\prime}, \varphi\right\rangle_{\Sigma}^{\prime}+\rho_{+}\left\langle\widetilde{w}_{+}^{\prime}, \psi\right\rangle_{\Omega+}^{\prime}+\rho_{-}\left\langle\widetilde{w}_{+}^{\prime}, \psi\right\rangle_{\Sigma}^{\prime} \\
+ & \rho_{+} b_{+}\left(\left(\widetilde{w}_{+}\right)^{\prime}, \psi\right)^{\prime}+\rho_{-} b_{\Sigma}\left(\left(\widetilde{w}_{+}\right)^{\prime}, \psi\right)^{\prime}+a_{+}\left(\widetilde{w}_{+}, \psi\right)+a_{\Sigma}\left(\widetilde{w}_{+}, \psi\right) \\
+ & \lambda_{+} c_{+}\left(\tilde{\phi}_{+}, \varphi\right)+\lambda_{-} c_{\Sigma}\left(\tilde{\phi}_{+}, \varphi\right)-\lambda_{+} b_{+}\left(\tilde{\theta}_{+}, \psi\right)-\lambda_{-} \tilde{b}_{\Sigma}\left(\tilde{\theta}_{+}, \psi\right) \\
+ & \rho_{+}\left\langle\tilde{\phi}_{+}, \zeta\right\rangle_{\Omega+}^{\prime}+\rho_{-}\left\langle\tilde{\phi}_{+}, \zeta\right\rangle_{\Sigma}^{\prime}+k_{+} b_{+}\left(\tilde{\phi}_{+}, \zeta\right)+k_{-} \bar{b}_{\Sigma}\left(\tilde{\phi}_{+}, \zeta\right) \\
- & \lambda_{+} d_{+}\left(\tilde{u}_{+}^{\prime}, \zeta\right)-\lambda_{-} d_{\Sigma}\left(\tilde{u}_{+}^{\prime}, \zeta\right)+\rho_{+}\left\langle\tilde{\theta}_{+}, \eta\right\rangle_{\Omega+}^{\prime}+\rho_{-}\left\langle\tilde{\theta}_{+}, \eta\right\rangle_{\Sigma}^{\prime} \\
+ & \lambda_{+} b_{+}\left(\left(\widetilde{w}_{+}\right)^{\prime}, \eta\right)+\lambda_{-} \bar{b}_{\Sigma}\left(\left(\widetilde{w}_{+}\right)^{\prime}, \eta\right)+k_{+} b_{+}\left(\tilde{\theta}_{+}, \eta\right)+k_{-} \bar{b}_{\Sigma}\left(\tilde{\theta}_{+}, \eta\right) \\
+ & N_{+}\left(\widetilde{u}_{+}, \widetilde{w}_{+}, \varphi_{+}, \psi_{+}\right)+N_{\Sigma}\left(\widetilde{u}_{+}, \widetilde{w}_{+}, \varphi, \psi\right)=0,
\end{aligned}
$$

$\forall(\varphi, \psi, \zeta, \eta) \in U\left(\Omega_{+}\right) \times W\left(\Omega_{+}\right) \times V\left(\Omega_{+}\right) \times V\left(\Omega_{+}\right)$, with the initial conditions

$$
\begin{aligned}
& \left.\tilde{u}_{+}(0)=u_{+}^{*}, \tilde{u}_{+}\right)^{\prime}(0)=u_{+}^{* *}, \tilde{w}_{+}(0)=w_{+}^{*},\left(\tilde{w}_{+}\right)^{\prime}(0)=w_{+}^{* *}, \tilde{\phi}_{+}(0)=\phi_{+}^{*}, \\
& \tilde{\theta}_{+}(0)=\theta_{+}^{*}, \quad \text { in } \Omega_{+} \\
& \tilde{w}_{+}(0)=w_{+\mid \Sigma}^{*},\left(\tilde{w}_{+}\right)^{\prime}(0)=w_{+\mid \Sigma}^{* *}, \tilde{u}_{+}(0)=u_{+\mid \Sigma}^{*},\left(\tilde{u}_{+}\right)^{\prime}(0)=u_{+}^{* * *}, \text { on } \Sigma, \\
& \left(\partial_{\nu} \tilde{w}_{+}\right)^{\prime}(0)=w_{+}^{* *}, \quad \tilde{\phi}_{+}(0)=\phi_{+\mid \Sigma}^{*}, \quad \tilde{\theta}_{+}(0)=\theta_{+\mid \Sigma}^{*} \quad \text { on } \Sigma,
\end{aligned}
$$

where

$$
\begin{aligned}
& a_{\Sigma}\left(\widetilde{w}_{+}, \psi\right)=\int_{\Sigma}\left[E_{-}\left(\gamma_{T}\left(\widetilde{w}_{+}\right) \gamma_{T}(\psi)+\frac{2}{\left(1+\mu_{-}\right)} \gamma_{S}\left(\widetilde{w}_{+}\right) \gamma_{S}(\psi)\right)\right] d s \\
& b_{\Sigma}\left(\widetilde{w}_{+}, \psi\right)=\int_{\Sigma}\left(\partial_{s} \widetilde{w}_{+} \partial_{s} \psi+\partial_{\nu} \widetilde{w}_{+} \partial_{\nu} \psi\right) d s, \quad \bar{b}_{\Sigma}\left(\tilde{\phi}_{+}, \zeta\right)=\int_{\Sigma} \partial_{s} \tilde{\phi}_{+} \partial_{s} \zeta d s, \\
& c_{\Sigma}\left(\tilde{\phi}_{+}, \varphi\right)=\int_{\Sigma}\left(-\partial_{s} \tilde{\phi}_{+} \varphi_{\tau}+\frac{\lambda_{-}}{k_{-}}\left(\widetilde{u}_{\nu}\right)_{+}^{\prime} \varphi_{\nu}\right) d s, \quad d_{\Sigma}\left(\tilde{u}_{+}^{\prime}, \zeta\right)=\int_{\Sigma}-\left(\widetilde{u}_{\tau}\right)_{+}^{\prime} \partial_{s} \zeta d s,
\end{aligned}
$$




$$
\begin{aligned}
& \tilde{b}_{\Sigma}\left(\widetilde{\theta}_{+}, \psi\right)=\int_{\Sigma}\left(\partial_{s} \widetilde{\theta}_{+} \partial_{s} \psi-\frac{\lambda_{-}}{k_{-}}\left(\partial_{\nu} \widetilde{w}_{+}\right)^{\prime} \partial_{\nu} \psi\right) d s, \\
& N_{\Sigma}\left(\widetilde{u}_{+}, \widetilde{w}_{+}, \varphi, \psi\right)=E_{-} \int_{\Sigma} N_{T}\left(\widetilde{u}_{+}, \widetilde{w}_{+}\right)\left(\partial_{s} \varphi_{\tau}-R(s) \varphi_{\nu}+\partial_{s} \widetilde{w}_{+} \partial_{s} \psi\right) d s .
\end{aligned}
$$

Proof. Let $\psi_{+} \in W\left(\Omega_{+}\right), \varphi_{+} \in U\left(\Omega_{+}\right), \quad \zeta \in V\left(\Omega_{+}\right)$and $\eta \in V\left(\Omega_{+}\right)$.We suppose that $\partial_{s} \psi_{+}$is bounded independently of $\delta$ in $H^{1}(\Sigma)$. We apply the variational problem (3.2) with the test functions

$$
\begin{array}{ll}
\psi= \begin{cases}\psi_{+} & \text {in } \Omega_{+}, \\
\psi_{+\mid \Sigma}+\delta z \partial_{\nu} \psi_{+} & \text {in } \Omega_{-},\end{cases} & \varphi= \begin{cases}\varphi_{+} & \text {in } \Omega_{+}, \\
\left(\varphi_{+\tau}, \delta \varphi_{+\nu}\right) & \text { in } \Omega_{-},\end{cases} \\
\zeta=\left\{\begin{array}{lll}
\zeta_{+} & \text {in } \Omega_{+}, \\
\zeta_{+\mid \Sigma} & \text { in } \Omega_{-}, & \eta
\end{array}\right. & \eta \begin{cases}\eta_{+} & \text {in } \Omega_{+}, \\
\eta_{+\mid \Sigma} & \text { in } \Omega_{-} .\end{cases}
\end{array}
$$

The same arguments as in the proof of Proposition 4 in [10] yield that

$$
\begin{aligned}
& b_{-}^{\delta}\left(\left(w_{-}^{\delta}\right)^{\prime}, \psi\right) \rightarrow b_{\Sigma}\left(\left(\widetilde{w}_{+}\right)^{\prime}, \psi_{+}\right), a_{-}^{\delta}\left(w_{-}^{\delta}, \psi\right) \rightarrow a_{\Sigma}\left(\widetilde{w}_{+}, \psi_{+}\right), \\
& N_{+}\left(u_{+}^{\delta}, w_{+}^{\delta}, \varphi_{+}, \psi_{+}\right) \rightarrow N_{+}\left(\widetilde{u}_{+}, \widetilde{w}_{+}, \varphi_{+}, \psi_{+}\right) \text {in } D^{\prime}(0, T) \text { as } \delta \rightarrow 0 .
\end{aligned}
$$

Moreover, taking advantage of Theorem 3, we can easily show that $c_{-}^{\delta}\left(\phi_{-}^{\delta}, \varphi_{-}\right)$ converges to $c_{\Sigma}\left(\tilde{\phi}_{+}, \varphi_{+}\right)$in $D^{\prime}(0, T)$ as $\delta \rightarrow 0$. Indeed, multiplying $c_{-}^{\delta}\left(\phi_{-}^{\delta}, \varphi_{-}\right)$ by $\xi \in D(0, T)$, integrating from 0 to $T$ and recalling the fact that $\int_{0}^{1} \frac{1}{\delta} \partial_{z} \phi_{-}^{\delta}(1-$ $R \delta z) d z \rightarrow \frac{\lambda_{-}}{k_{-}}\left(\widetilde{u}_{+\nu}\right)^{\prime}$ weakly in $L^{2}\left(0, T ; L^{2}(\Sigma)\right)$, we obtain

$$
\begin{aligned}
& \int_{0}^{T} \int_{\Sigma} \int_{0}^{1}\left(-(1-R \delta z)^{-1} \partial_{s} \phi_{-}^{\delta} \varphi_{-\tau}+\delta^{-2} \partial_{z} \phi_{-}^{\delta} \varphi_{-\nu}\right)(1-R \delta z) \xi(t) d s d z \\
& \rightarrow \int_{0}^{T} \int_{\Sigma}\left(-\partial_{s} \widetilde{\phi}_{+} \varphi_{+\tau}+\frac{\lambda_{-}}{k_{-}}\left(\widetilde{u}_{\nu}\right)_{+}^{\prime} \varphi_{+\nu}\right) \xi(t) d s d t .
\end{aligned}
$$

The convergence above follows from the definition of the weak convergence in $L^{2}\left(0, T ; L^{2}(\Sigma)\right)$ : note that $\xi \varphi_{-\nu}$ and $\xi \varphi_{-\tau}$ belong to the space $L^{2}\left(0, T ; L^{2}(\Sigma)\right)$ and that $\int_{0}^{1} \partial_{s} \phi_{-}^{\delta} d z \rightarrow \partial_{s} \widetilde{\phi}_{+}$weakly in $L^{2}\left(0, T ; L^{2}(\Sigma)\right)$. Arguing as before and using the two last limits of Theorem 3 , we obtain the convergence of $d_{-}^{\delta}\left(\left(u_{-}^{\delta}\right)^{\prime}, \zeta_{-}\right), b_{-}^{\delta}\left(\theta_{-}^{\delta}, \psi_{-}\right), b_{-}^{\delta}\left(\left(w_{-}^{\delta}\right)^{\prime}, \eta_{-}\right), b_{-}^{\delta}\left(\theta_{-}^{\delta}, \eta_{-}\right)$and $b_{-}^{\delta}\left(\phi_{-}^{\delta}, \zeta_{-}\right)$towards $d_{\Sigma}\left(\tilde{u}_{+}^{\prime}, \zeta\right), \overline{\tilde{b}}_{\Sigma}\left(\widetilde{\theta}_{+}, \psi\right), \bar{b}_{\Sigma}\left(\left(\widetilde{w}_{+}\right)^{\prime}, \eta\right), \bar{b}_{\Sigma}\left(\tilde{\theta}_{+}, \eta\right)$ and $\bar{b}_{\Sigma}\left(\tilde{\phi}_{+}, \zeta\right)$, respectively, in $D^{\prime}(0, T)$.

Finally, we show that $N_{-}^{\delta}\left(u_{-}^{\delta}, w_{-}^{\delta}, \varphi_{-}, \psi_{-}\right) \rightarrow N_{\Sigma}\left(\widetilde{u}_{+}, \widetilde{w}_{+}, \varphi_{+}, \psi_{+}\right)$as in the proof of Proposition 4 in [10]. The difficulties arising from the nonlinear terms are treated thanks to the Sobolev imbeddings and the a priori estimates proved 
in Theorem 1. To illustrate this (see [10] for the details), we have for instance:

$$
\begin{aligned}
& \mid \int_{0}^{T} \int_{\Sigma} \int_{0}^{1}\left\{\left[N_{T}^{\delta}\left(u_{-}^{\delta}, w_{-}^{\delta}\right)+\frac{\mu_{-}}{\delta^{2}} N_{N}^{\delta}\left(u_{-}^{\delta}, w_{-}^{\delta}\right)\right]\left[\frac{1}{(1-R \delta z)^{2}} \partial_{s} w_{-}^{\delta} \partial_{s} \psi_{-}\right](1-R \delta z)\right. \\
& \left.\quad-\left(1-\mu_{-}\right) N_{T}\left(\widetilde{u}_{+}, \widetilde{w}_{+}\right) \partial_{s} \widetilde{w}_{+} \partial_{s} \psi_{+}\right\} \xi(t) d s d z d t|\leq| \int_{0}^{T} \int_{\Sigma} \int_{0}^{1}\left[\left(N_{T}^{\delta}\left(u_{-}^{\delta}, w_{-}^{\delta}\right)\right.\right. \\
& \left.\left.\quad+\frac{\mu_{-}}{\delta^{2}} N_{N}^{\delta}\left(u_{-}^{\delta}, w_{-}^{\delta}\right)\right)(1-R \delta z)-\left(1-\mu_{-}\right) N_{T}\left(\widetilde{u}_{+}, \widetilde{w}_{+}\right)\right] \partial_{s} \widetilde{w}_{+} \partial_{s} \psi_{+} \xi(t) d s d z d t \mid \\
& \quad+\int_{0}^{T}\left\|\left(N_{T}^{\delta}\left(u_{-}^{\delta}, w_{-}^{\delta}\right)+\mu_{-} \delta^{-2} N_{N}^{\delta}\left(u^{\delta}, w^{\delta}\right)\right)\right\|_{L^{2}}\left\|\partial_{s} w_{-}^{\delta}\right\| L_{L^{3}} \|(1-R \delta z)^{-2} \partial_{s} \psi_{-}^{\delta} \\
& \quad-\partial_{s} \psi_{+}\left\|_{L^{6}}|\xi(t)| d t+\int_{0}^{T}\right\|\left(N_{T}^{\delta}\left(u_{-}^{\delta}, w_{-}^{\delta}\right)+\mu_{-} \delta^{-2} N_{N}^{\delta}\left(u_{-}^{\delta}, w_{-}^{\delta}\right)\right) \|_{L^{2}\left(\Omega_{-}\right)} \\
& \quad \times\left\|\partial_{s} \psi_{+}\right\|_{L^{3}\left(\Omega_{-}\right)}\left\|\partial_{s} w_{-}^{\delta}-\partial_{s} \widetilde{w}_{+}\right\|_{L^{6}}|\xi(t)| d t,
\end{aligned}
$$

where $\xi \in D(0, T)$. The first integral in the right-hand side of the above inequality converges to 0 as $\delta \rightarrow 0$, by definition of the weak ${ }^{*}$ convergence (note that $\partial_{s} \widetilde{w}_{+} \partial_{z} \psi_{+} \xi$ is in $L^{1}\left(0, T ; L^{2}(\Sigma)\right.$ ).

Using a compactness we obtain that $\partial_{s} w_{-}^{\delta} \rightarrow \partial_{s} \widetilde{w}_{+}$in $L^{\infty}\left(0, T ; H^{1-\varepsilon}(\Omega)\right)$ strongly $\forall \varepsilon>0$. Since in two space dimensions we have the imbedding $H^{1-\varepsilon}(\Omega) \subset L^{\frac{2}{\varepsilon}}(\Omega)$, we deduce that for $\varepsilon$ small enough, $\partial_{s} w_{-}^{\delta} \rightarrow \partial_{s} \widetilde{w}_{+}$strongly in $L^{\infty}\left(0, T ; L^{6}\left(\Omega_{-}\right)\right)$. Thus, using the a priori estimates proved in Theorem 1 , we conclude that the second and the third integrals in the right hand side of the last inequality converge to zero as $\delta \rightarrow 0$. Combining the above, we obtain the desired result. Recalling the above results and going to the limit in (3.2) and the initial data, we obtain the Cauchy-Ventcel problem.

Existence of a weak solution can be shown by means of Faedo-Galerkin approximation. However, the uniqueness issue is much more delicate (see the remark following the proof of Proposition 4 in [10]). We also refer to [3] and [7], which may be applied to the limit problem.

Remark 4. The limit problem obtained above is, at least formally, equivalent to the following boundary value problem

$$
\begin{aligned}
& \rho_{+}\left(\widetilde{u}_{+}\right)^{\prime \prime}-\operatorname{div}\left\{C\left[\epsilon\left(\widetilde{u}_{+}\right)+f\left(\nabla \widetilde{w}_{+}\right)\right]\right\}+\lambda_{+} \nabla \tilde{\phi}_{+}=0 \quad \operatorname{in} \Omega_{+} \times(0, T), \\
& \rho_{+}[I-\Delta] \widetilde{w}_{+}^{\prime \prime}+D_{+} \Delta^{2} \widetilde{w}_{+}-\operatorname{div}\left\{C\left[\epsilon\left(\widetilde{u}_{+}\right)+f\left(\nabla \widetilde{w}_{+}\right)\right] \nabla \widetilde{w}_{+}\right\}+\lambda_{+} \Delta \tilde{\theta}_{+}=0
\end{aligned}
$$

in $\Omega_{+} \times(0, T)$ with Dirichlet conditions given on $\Gamma_{+}$

$$
\widetilde{u}_{+}=0, \widetilde{w}_{+}=\partial_{\nu} \widetilde{w}_{+}=0, \tilde{\theta}_{+}=0, \tilde{\phi}_{+}=0 \text { on } \Gamma_{+} \times(0, T)
$$

and the Ventcel's conditions on $\Sigma \times(0, T)$ :

$$
{ }^{t} \tau\left(C\left[\epsilon\left(\widetilde{u}_{+}\right)+f\left(\nabla \widetilde{w}_{+}\right)\right]\right) \nu=-\rho_{-}\left(\widetilde{u}_{\tau}\right)_{+}^{\prime \prime}+E_{-} \partial_{s}\left[N_{T}\left(\widetilde{u}_{+}, \widetilde{w}_{+}\right)\right]+\lambda_{-} \partial_{s} \tilde{\phi}_{+},
$$




$$
\begin{aligned}
& { }^{t} \nu\left(C\left[\epsilon\left(\widetilde{u}_{+}\right)+f\left(\nabla \widetilde{w}_{+}\right)\right]\right) \nu=-\rho_{-}\left(\widetilde{u}_{\nu}\right)_{+}^{\prime \prime}+E_{-} R(s) N_{T}\left(\widetilde{u}_{+}, \widetilde{w}_{+}\right)-\lambda_{-}^{2}\left(\widetilde{u}_{\nu}\right)_{+}^{\prime} / k_{-}, \\
& D_{+}\left[\Delta \widetilde{w}_{+}+(1-\mu) B_{1} \widetilde{w}_{+}\right]=-\left(Q\left(\widetilde{w}_{+}\right)+\rho_{-} \partial_{\nu} \widetilde{w}_{+}^{\prime \prime}+\lambda_{-}^{2}\left(\partial_{\nu} \widetilde{w}_{+}\right)^{\prime}\right) / k_{-}, \\
& D_{+}\left[\partial_{\nu} \Delta \widetilde{w}_{+}+\left(1-\mu_{+}\right) \partial_{s} B_{2} \widetilde{w}_{+}\right]-\rho_{+} \partial_{\nu} \widetilde{w}_{+}^{\prime \prime}-C\left[\epsilon\left(\widetilde{u}_{+}\right)+f\left(\nabla \widetilde{w}_{+}\right)\right] \nu \nabla \widetilde{w}_{+} \\
& \quad+\lambda_{+} \partial_{\nu} \tilde{\theta}_{+}=\rho_{-}\left[\widetilde{w}_{+}-\partial_{s}^{2} \widetilde{w}_{+}\right]^{\prime \prime}+P\left(\widetilde{w}_{+}\right)-E_{-} \partial_{s}\left[N_{T}\left(\widetilde{u}_{+}, \widetilde{w}_{+}\right) \partial_{s} \widetilde{w}_{+}\right]+\lambda_{-} \partial_{s}^{2} \tilde{\theta}_{+}, \\
& k_{+} \partial_{\nu} \tilde{\theta}_{+}+\lambda_{+} \partial_{\nu} \tilde{w}^{\prime}=-\rho_{-} \tilde{\theta}_{+}^{\prime}+k_{-} \partial_{s}^{2} \tilde{\theta}_{+}+\lambda_{-} \partial_{s}^{2} \tilde{w}_{+}^{\prime}, \\
& k_{+} \partial_{\nu} \tilde{\phi}_{+}-\lambda_{+} \tilde{u}_{+}^{\prime} \nu=-\rho_{-} \tilde{\phi}_{+}^{\prime}+k_{-} \partial_{s}^{2} \tilde{\phi}_{+}+\lambda_{-} \partial_{s}\left(\tilde{u}_{\tau}\right)_{+}^{\prime},
\end{aligned}
$$

where ${ }^{t} \nu$ (resp. $\left.{ }^{t} \tau\right)$ is the transposed vector of $\nu$ (resp. $\tau$ ).

With the system given above, we associate the initial conditions

$$
\begin{aligned}
& \tilde{u}_{+}(0)=u_{+}^{*}, \quad\left(\tilde{u}_{+}\right)^{\prime}(0)=u_{+}^{* *}, \tilde{w}_{+}(0)=w_{+}^{*}, \quad\left(\tilde{w}_{+}\right)^{\prime}(0)=w_{+}^{* *}, \\
& \tilde{\phi}_{+}(0)=\phi_{+}^{*}, \quad \tilde{\theta}_{+}(0)=\theta_{+}^{*}, \quad \operatorname{in} \Omega_{+} \\
& \tilde{w}_{+}(0)=w_{+\mid \Sigma}^{*}, \quad\left(\tilde{w}_{+}\right)^{\prime}(0)=\stackrel{w}{+} \mid \Sigma_{+}^{* *} \quad \tilde{u}_{+}(0)=u_{+\mid \Sigma}^{*}, \quad\left(\tilde{u}_{+}\right)^{\prime}(0)=u_{+}^{* *}, \quad \text { on } \Sigma, \\
& \left(\partial_{\nu} \tilde{w}_{+}\right)^{\prime}(0)=\stackrel{*}{w *}_{+}^{* *}, \quad \tilde{\phi}_{+}(0)=\phi_{+\mid \Sigma}^{*}, \quad \tilde{\theta}_{+}(0)=\theta_{+\mid \Sigma}^{*} \quad \text { on } \Sigma .
\end{aligned}
$$

The operators $P$ et $Q$ are defined by:

$$
\begin{aligned}
& P(\widetilde{w})=E_{-}\left[\partial_{s}^{2} \gamma_{T}(\widetilde{w})+2 \partial_{s}\left(R(s) \gamma_{S}(\widetilde{w})\right) /\left(1+\mu_{-}\right)\right], \\
& Q(\widetilde{w})=E_{-}\left[2 \partial_{s} \gamma_{S}(\widetilde{w}) /\left(1+\mu_{-}\right)-R(s) \gamma_{T}(\widetilde{w})\right] .
\end{aligned}
$$

We have thus obtained a new problem posed only over the set $\Omega_{+}$, which is nothing but the domain occupied by the plate. However, the effect of the stiffener is taken into account and is completely embodied by the additive terms that are involved in the right hand sides of the boundary conditions imposed along the portion of the boundary $\Sigma$, into which the stiffener was inserted. Indeed, one observes that these terms depend solely on the material constituting the stiffener, through its elastic and thermal characteristics $E_{-}$, $\mu_{-}, \rho_{-}, k_{-}$and $\lambda_{-}$. Moreover, the effect of the thin body is also expressed by means of the new initial conditions imposed on $\Sigma$.

Finally, let us mention, that the assumption made on the elastic and thermal characteristics of the material constituting the stiffener (they behave as $\delta^{-1}$ ) is of a major importance. It is crucial to observe that, these "relative" asymptotic orders need to be assumed in order to obtain the model described above and take into account the mechanical and thermal effect of the stiffener. Indeed, other ratios between the "asymptotic orders" in each substructure can lead to strikingly different limit behaviors. To illustrate this assertion, assume that both the plate's and stiffener's characteristics are independent of $\delta$. Thus, we obtain, as $\delta \rightarrow 0$, a problem where the effect of the thin stiffener is completely neglected. In this case, the model derived is simply obtained by omitting the thin body. As it can be seen in the results obtained in the present paper, a completely different limit behavior occurs if the characteristics of the inserted body approach $+\infty$ sufficiently rapidly as $\delta \rightarrow 0$. This comes from the fact that, in this situation, the material constituting the stiffener is more rigid and 
more conductive than that constituting the plate: the rigidity and the high thermal conductivity of the stiffener emphasize its effect on the displacement and the temperature of the plate.

We thus reach the interesting conclusion that, in order to get a "limit" problem that takes into account the effect of the stiffener, as its thickness goes to zero, it turns out to be sufficient to compensate this thickness by a specific increase as $\delta \rightarrow 0$, of the rigidity and the thermal conductivity of the material that constitutes this latter. The approximate boundary conditions obtained in this paper model the presence of the stiffener and express the influence of this latter on the oscillations and the propagation of heat inside the plate.

\section{References}

[1] H. Ammari and C. Latiri-Grouz. Approximate boundary conditions for thin periodic coatings. In Mathematical and numerical aspects of wave propagation (Golden, CO, 1998), pp. 297-301. SIAM, Philadelphia, PA, 1998.

[2] A. Andreev and S. Samarsky. Méthodes aux différences pour les équations elliptiques. Edition de Moscou, Moscou, 1978.

[3] A. Benabdallah and I. Lasiecka. Exponential decay rates for a full von karman system of dynamic thermoelasticity. Journal of Differential Equations, 160:5193, 2000.

[4] A. Bendali and K. Lemrabet. The effect of a thin coating on the scattering of a time-harmonic wave for the Helmholtz equation. SIAM J. Appl. Math., 56(6):1664-1693, 1996.

[5] B. Engquist and J-C. Nédélec. Effective boundary conditions for acoustic and electromagnetic scattering in thin layers. Rapport interne 278, CMAP, école polytechnique, Palaiseau, France, 1993.

[6] J.E. Lagnese and J.L. Lions. Modelling, Analysis and Control of Thin Plates. Masson, Paris, 1988.

[7] I. Lasiesca. Uniform decay rates for full von karman system of dynamic thermoelasticity with free boundary conditions and partial boundary dissipation. Partial Differential Equations, 24((9-10)):1801-1847, 1999.

[8] K. Lemrabet. Problèmes aux limites de ventcel dans un domaine non régulier. C.R. Acad. Sci. Paris. Série I, 300:531-534, 1985.

[9] K. Lemrabet. Etude de divers problèmes aux limites de ventcel d'origine physique ou mécanique dans des domaines non ré guliers. Thèse de Doctorat d'Etat, U.S.T.H.B, 1987.

[10] L.Rahmani. Ventcel's boundary conditions for a dynamic nonlinear plate. Asymptotic Analysis, 38:319-337, 2004.

[11] L. Rahmani. Conditions aux limites approchées pour une plaque mince non linéaire. C.R. Acad. Sci. Paris, Ser.I, 343:57-62, 2006.

[12] L. Rahmani. The effect of a thin layer on a thermoelastic nonlinear plate. Applied Mathematical science, 2(50):2489-2499, 2008.

[13] L. Rahmani and G. Vial. Rienforcement of a thin plate by a thin layer. Mathematical Methods in the Applied Sciences, 31:315-338, 2008. 